\title{
Hip Musculoskeletal Conditions and Associated Factors That Influence Squat Performance: A Systematic Review
}

\author{
Scott W. Cheatham, Kyle R. Stull, Mike Fantigrassi, and lan Montel
}

\begin{abstract}
Context: The squat is a fundamental movement for weightlifting and sports performance. Both unilateral and bilateral squats are also used to assess transitional and dynamic lower-extremity control. Common lower-extremity conditions can have an influence on squat performance. Of interest are the effects of hip musculoskeletal conditions and associated factors, such as hip muscle pain, fatigue, and tightness, on squat performance. Currently, there has been no appraisal of the evidence regarding the association of these conditions and associated factors on squat performance. Objective: This study evaluated the current evidence regarding common hip musculoskeletal conditions and associated factors, such as hip muscle pain, fatigue, and tightness, on squat performance. Evidence Acquisition: A systematic review was conducted according to preferred reporting items for systematic reviews and meta-analyses guidelines. A search of PubMed, CINAHL, SPORTDiscus, ProQuest, and Google Scholar ${ }^{\circledR}$ was conducted in October, 2016 using the following keywords alone and in combination: hip, joint, arthritis, pain, range of motion (ROM), fatigue, tightness, pathology, condition, muscle, intraarticular, extraarticular, femoroacetabular impingement, single leg, bilateral, squat, performance, and technique. The grading of studies was conducted using the Physiotherapy Evidence Database scale. Evidence Synthesis: The authors identified 35 citations, 15 of which met the inclusion criteria. The qualifying studies yielded a total of 542 subjects (160 men and 382 women; mean age $=29.3(5.9)$ y) and measured performance with either the barbell squat, step down, bilateral, or single-leg squat. Femoroacetabular impingement and hip arthroscopy were the only hip conditions found that affected the squat. Associated factors, such as muscle pain, fatigue, and tightness, also influenced squat performance. Conclusion: This review found that common hip conditions and associated factors and their effects on squat performance to be underinvestigated. Future research should focus on the association between common hip conditions and squat performance.
\end{abstract}

Keywords: pain, technique, femoroacetabular, joint, arthritis

The squat is a foundational exercise utilized in resistance training programs. Often, the squat is used to develop strength and power in the lower-extremity, and is a fundamental exercise in an athlete's training program. Allied health and fitness professionals often prescribe different versions of unilateral and bilateral squats to challenge clients in their training programs. Research has shown that both unilateral and bilateral squat training can improve lower body strength, sprint speed, and agility in individuals. ${ }^{1}$ Both unilateral and bilateral squats are also used as assessments of dynamic lower-extremity motor control. ${ }^{2-7}$ The utility of unilateral and bilateral squats for training and assessment has made these movements a foundational part of athletic and exercise training programs.

Common lower-extremity conditions can have an influence on the squat movement and must be considered when training and assessing injured athletes. Injuries to the hip have become an emerging area of research due to improved recognition of hip conditions and the advent of hip arthroscopy. ${ }^{8}$ Statistics have estimated that $10 \%$ to $20 \%$ of athletic or recreation childhood injuries are hip related. ${ }^{9-11}$ Also, $5 \%$ to $6 \%$ of adult sports injuries occur at the hip and pelvis. ${ }^{9-11}$ It is important for allied health and fitness professionals to be able to recognize common hip musculoskeletal conditions and how they can affect movements, such as the squat.

Cheatham is with the California State University Dominguez Hills, Carson, CA. Stull, Fantigrassi, and Montel are with the National Academy of Sports Medicine, Chandler, AZ. Cheatham (Scheatham@csudh.edu) is corresponding author.
Of interest are the effects of common hip musculoskeletal conditions on squat performance. Other factors, such as increased hip muscle pain, fatigue, and tightness, are also of interest as they often accompany hip joint conditions and can affect exercise performance. ${ }^{8,12-15}$ A large body of evidence exists that associates hip muscle weakness with poor bilateral ${ }^{16-20}$ and single-leg squat ${ }^{21-23}$ performance. To date, there has been no appraisal of the evidence regarding the association of hip musculoskeletal conditions or associated factors and squat performance. Such an appraisal may help guide allied health and fitness professionals when designing an exercise program for these clients. The purpose of this systematic review was to appraise the evidence regarding the effects of hip musculoskeletal conditions and associated factors, such as hip muscle pain, fatigue, and tightness, on squat performance.

\section{Methods}

\section{Search Strategy}

A systematic review was conducted according the preferred reporting items for systematic reviews and meta-analyses guidelines for reporting systematic reviews. ${ }^{24,25}$ Relevant articles, through October, 2016, were identified via independent searches of electronic databases that included PubMed, CINAHL, SPORTDiscus, ProQuest, and Google Scholar®. A direct search of known journals was also conducted to identify potential publications. For this review, hip musculoskeletal conditions were defined as commonly diagnosed intraarticular and extraarticular conditions. Hip joint 
replacement and muscle weakness were not included in this analysis. The search terms used included individual or a combination of the following: hip, joint, arthritis, pain, range of motion (ROM), fatigue, tightness, pathology, condition, muscle, intraarticular, extraarticular, femoroacetabular impingement, single leg, bilateral, squat, performance, and technique.

\section{Study Selection}

Two reviewers independently evaluated eligible manuscripts identified through the search strategy outlined in the previous section. A third independent reviewer was available to resolve any disagreements. Studies considered for inclusion met the following criteria: (1) peer-reviewed, English-language publications and (2) controlled clinical trials that examined the relationship between hip conditions and associated factors with the squat. Excluded studies included the following: non-English publications, clinical trials that examined hip pathologies and/or associated factors but did not directly measure their effects on the squat performance, clinical trials involving total joint replacement or hip muscle weakness, case reports, case series, clinical commentary, dissertations, and conference posters or abstracts.

\section{Data Extraction and Synthesis}

The following data were extracted from each article: subject demographics, hip conditions, movement, outcomes, and results. The research design of each study was also identified by the reviewers. Qualifying manuscripts were assessed according to the Physiotherapy Evidence Database scale for appraising the quality of literature. ${ }^{26,27}$ Interobserver agreement was calculated using the kappa statistic. ${ }^{28}$ Reasons for exclusion of manuscripts are outlined in Figure 1.

\section{Results}

The interobserver agreement was substantial $\kappa=0.90$ for the title selection and substantial $\kappa=0.93$ for abstract selection. The

Search strategy

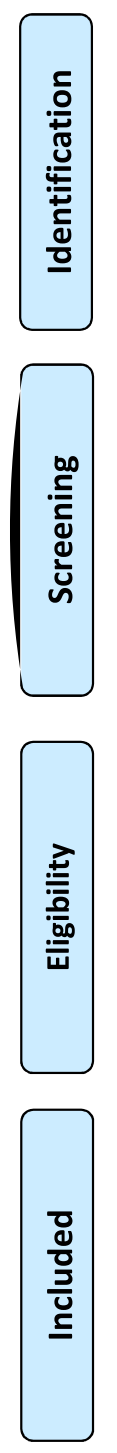

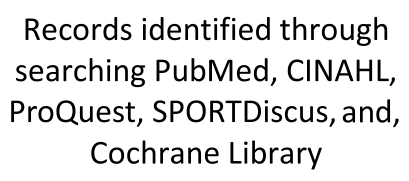
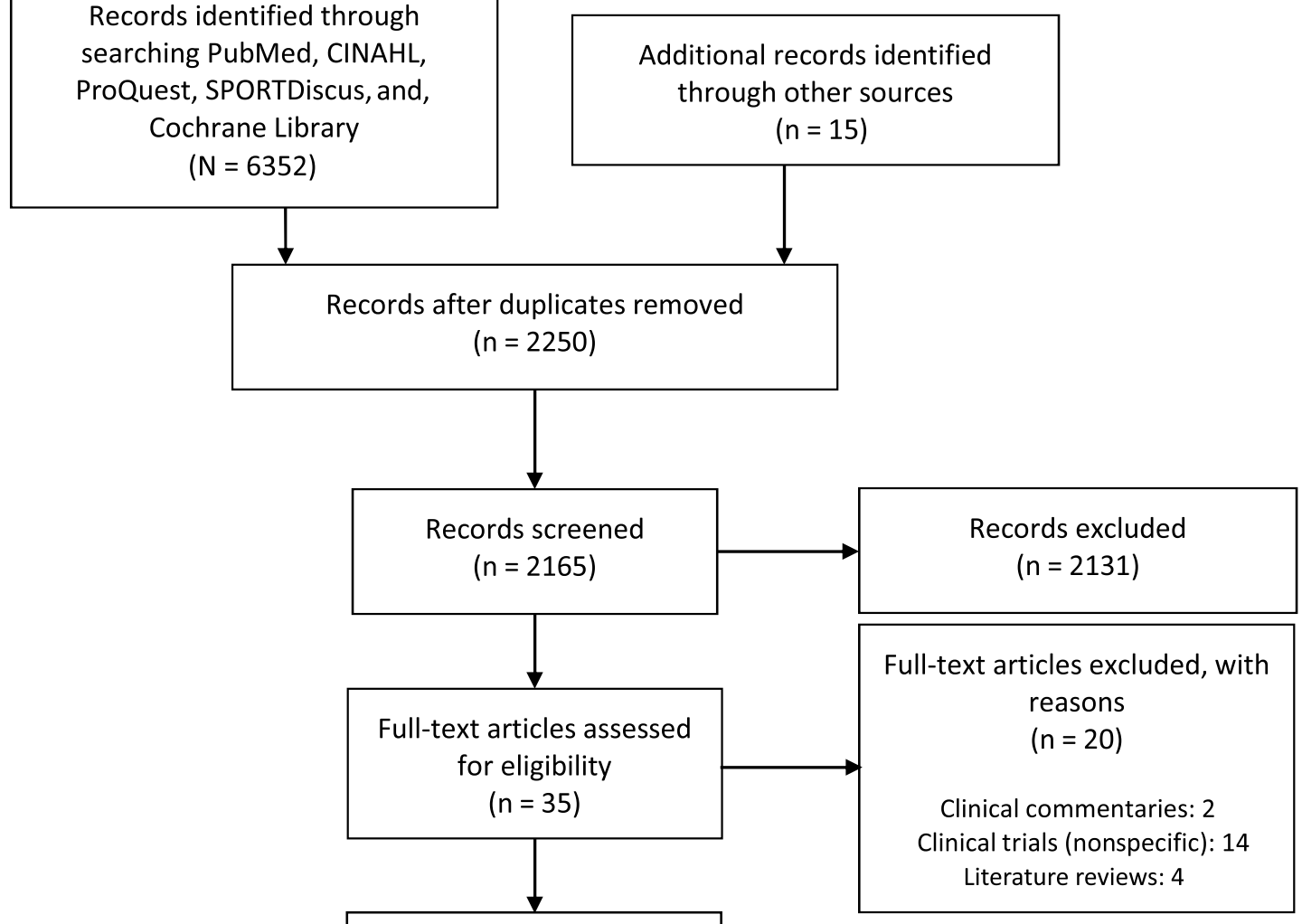

Studies included in

qualitative synthesis

$(n=15)$

Figure 1 - PRISMA search strategy. PRISMA indicates preferred reporting items for systematic reviews and meta-analyses. 
reviewers identified a total of 6352 publications from electronic databases, and 15 publications were identified through direct searches for a total of 6367 manuscripts (Figure 1). Thirty-five articles qualified for the full-text search, and 20 articles were excluded (Figure 1). Fifteen articles met the inclusion criteria for the evidence synthesis and were graded with the Physiotherapy Evidence Database scale (Table 1). Three studies measured the effects of femoroacetabular impingement (FAI), ${ }^{15,24,25} 1$ study measured the effects of hip chondropathy (HC), ${ }^{28}$ and 2 studies measured the effects of hip arthroscopy surgery ${ }^{14,26}$ on squat performance (Table 2). Two studies examined the effects of muscle pain, ${ }^{27,29} 5$ studies measured the effects of muscle fatigue, ${ }^{30-34}$ and 2 studies reported the effects of muscle tightness ${ }^{35,36}$ on squat performance (Table 3). All qualifying manuscripts yielded a total of 542 subjects (160 men and 382 women; mean age $=29.3(5.9)$ y) and measured performance with either the barbell squat, step down, bilateral, or single-leg squat. ${ }^{14,15,24-36}$ Several studies also included other measures of performance that are described in Tables 2 and 3.24,28,29,33,36 The following sections discuss the results of this analysis.

\section{Femoroacetabular Impingement}

Emerging research has demonstrated that FAI can affect squat performance due to the abnormal contact between the acetabular rim and femoral head-neck junction resulting from abnormal bone growth. ${ }^{12}$ There are 3 common types of FAI based on where the abnormal bone growth has occurred-along the acetabular rim (FAI-Pincer), at the femoral head-neck junction (FAI-CAM), or both (FAI-CAM/Pincer) ${ }^{8,12}$ Bagwell et al $^{15}$ examined hip and pelvic kinematics in a group of 30 participants (mean age $=32$ (7.7) y) assigned to 2 groups. Group 1 consisted of 15 subjects (6 men and 9 women) diagnosed with FAI-CAM impingement and group 2 consisted of 15 matched asymptomatic subjects ( 6 men and 9 women). The researcher analyzed peak hip flexion, abduction, and internal rotation ROM, and calculated mean hip extensor, adductor, and external rotator moments during a bodyweight squat. Upon completion, the researchers found that subjects with FAI had decreased hip internal rotation $\operatorname{ROM}(P=.04)$, decreased hipextensor moments $(P=.02)$, and a more anteriorly tilted pelvis $(P=.02)$ when compared with the control group. ${ }^{15}$ Lamontagne et $\mathrm{al}^{25}$ also analyzed hip and pelvic kinematics during a bodyweight maximal deep squat movement in a group of 32 male subjects (mean age $=34.9(9.5) \mathrm{y})$. Group 1 consisted of 15 subjects diagnosed with FAI-CAM and group 2 consisted of 11 matched healthy control subjects. The researcher analyzed hip and pelvic motion using 3dimensional (3D) analysis. Upon completion, the researchers found that subjects with FAI had no difference in hip motion but had decreased sagittal plane pelvic ROM throughout the movement and a decreased squat depth when compared with the control group. ${ }^{25}$

Kumar et $\mathrm{al}^{24}$ also investigated hip movement pattern in a group of 15 subjects (mean age $=31.6(9.7)$ y). Group 1 consisted of 7 subjects ( 5 men and 2 women) diagnosed with unilateral FAICAM hip impingement and group 2 consisted of 8 healthy control subjects (8 men). The researchers also conducted a secondary analysis using the same subjects in which they compared the male subjects with FAI-CAM and cartilage lesions $(\mathrm{N}=6)$ and subjects without cartilage lesions $(\mathrm{N}=9)$. The researchers analyzed the subjects' performance during walking, squatting, and drop landing using 3D motion analysis. Upon completion, the researchers found that subjects with FAI-CAM and FAI-CAM with cartilage lesions demonstrated reduced performance on the squat movement. Subjects also demonstrated increased hip adduction and internal rotation, and decreased transverse plane ROM during the squat when compared with controls. Increased hip adduction and internal rotation were also seen in subjects with FAI-CAM (with and without cartilage lesions) during the drop landing task when compared with controls. No difference was found between groups for walking. ${ }^{24}$

Table 1 PEDro Scores for Qualified Studies

\begin{tabular}{|c|c|c|c|c|c|c|c|c|c|c|c|c|}
\hline & Item 1 & Item 2 & Item 3 & Item 4 & Item 5 & Item 6 & Item 7 & Item 8 & Item 9 & Item 10 & Item 11 & Total score \\
\hline Hatton et $\mathrm{al}^{27}$ & $\mathrm{Y}$ & $\mathrm{N}$ & $\mathrm{N}$ & $\mathrm{Y}$ & $\mathrm{N}$ & $\mathrm{N}$ & $\mathrm{N}$ & $\mathrm{Y}$ & $\mathrm{Y}$ & $\mathrm{Y}$ & $\mathrm{Y}$ & 6 \\
\hline Hatton et $\mathrm{al}^{29}$ & $\mathrm{Y}$ & $\mathrm{N}$ & $\mathrm{N}$ & $\mathrm{Y}$ & $\mathrm{N}$ & $\mathrm{N}$ & $\mathrm{N}$ & $\mathrm{Y}$ & $\mathrm{Y}$ & $\mathrm{Y}$ & $\mathrm{Y}$ & 6 \\
\hline Hooper et $\mathrm{al}^{32}$ & $\mathrm{Y}$ & $\mathrm{N}$ & $\mathrm{N}$ & $\mathrm{Y}$ & $\mathrm{N}$ & $\mathrm{N}$ & $\mathrm{N}$ & $\mathrm{Y}$ & $\mathrm{Y}$ & $\mathrm{N}$ & $\mathrm{Y}$ & 5 \\
\hline Hooper et $\mathrm{al}^{31}$ & $\mathrm{Y}$ & $\mathrm{N}$ & $\mathrm{N}$ & $\mathrm{Y}$ & $\mathrm{N}$ & $\mathrm{N}$ & $\mathrm{N}$ & $\mathrm{Y}$ & $\mathrm{Y}$ & $\mathrm{N}$ & $\mathrm{Y}$ & 5 \\
\hline Weeks et $\mathrm{al}^{34}$ & $\mathrm{Y}$ & $\mathrm{N}$ & $\mathrm{N}$ & $\mathrm{Y}$ & $\mathrm{N}$ & $\mathrm{N}$ & $\mathrm{N}$ & $\mathrm{Y}$ & $\mathrm{Y}$ & $\mathrm{N}$ & $\mathrm{Y}$ & 5 \\
\hline McMullen et $\mathrm{al}^{33}$ & $\mathrm{Y}$ & $\mathrm{N}$ & $\mathrm{N}$ & $\mathrm{Y}$ & $\mathrm{N}$ & $\mathrm{N}$ & $\mathrm{N}$ & $\mathrm{Y}$ & $\mathrm{Y}$ & $\mathrm{N}$ & $\mathrm{Y}$ & 5 \\
\hline Hodges et $\mathrm{al}^{30}$ & $\mathrm{Y}$ & $\mathrm{N}$ & $\mathrm{N}$ & $\mathrm{Y}$ & $\mathrm{N}$ & $\mathrm{N}$ & $\mathrm{N}$ & $\mathrm{Y}$ & Y & $\mathrm{N}$ & $\mathrm{Y}$ & 5 \\
\hline Mills et $\mathrm{al}^{35}$ & $\mathrm{Y}$ & $\mathrm{N}$ & $\mathrm{N}$ & $\mathrm{Y}$ & $\mathrm{N}$ & $\mathrm{N}$ & $\mathrm{N}$ & $\mathrm{Y}$ & Y & $\mathrm{Y}$ & $\mathrm{Y}$ & 6 \\
\hline Bagwell et al ${ }^{15}$ & $\mathrm{Y}$ & $\mathrm{N}$ & $\mathrm{N}$ & $\mathrm{Y}$ & $\mathrm{N}$ & $\mathrm{N}$ & $\mathrm{N}$ & $\mathrm{Y}$ & Y & $\mathrm{Y}$ & $\mathrm{Y}$ & 6 \\
\hline Lamontagne et $\mathrm{al}^{25}$ & $\mathrm{Y}$ & $\mathrm{N}$ & $\mathrm{N}$ & $\mathrm{Y}$ & $\mathrm{N}$ & $\mathrm{N}$ & $\mathrm{N}$ & $\mathrm{Y}$ & $\mathrm{Y}$ & $\mathrm{Y}$ & $\mathrm{Y}$ & 6 \\
\hline Kumar et $\mathrm{al}^{24}$ & $\mathrm{Y}$ & $\mathrm{N}$ & $\mathrm{N}$ & $\mathrm{Y}$ & $\mathrm{N}$ & $\mathrm{N}$ & $\mathrm{N}$ & $\mathrm{Y}$ & $\mathrm{Y}$ & $\mathrm{Y}$ & $\mathrm{Y}$ & 6 \\
\hline Hatton et $\mathrm{al}^{28}$ & $\mathrm{Y}$ & $\mathrm{N}$ & $\mathrm{N}$ & $\mathrm{Y}$ & $\mathrm{N}$ & $\mathrm{N}$ & $\mathrm{N}$ & $\mathrm{Y}$ & $\mathrm{Y}$ & $\mathrm{Y}$ & $\mathrm{Y}$ & 6 \\
\hline Charlton et al ${ }^{14}$ & $\mathrm{Y}$ & $\mathrm{N}$ & $\mathrm{N}$ & $\mathrm{Y}$ & $\mathrm{N}$ & $\mathrm{N}$ & $\mathrm{N}$ & $\mathrm{Y}$ & $\mathrm{Y}$ & $\mathrm{Y}$ & $\mathrm{Y}$ & 6 \\
\hline Lamontagne et $\mathrm{al}^{26}$ & $\mathrm{Y}$ & $\mathrm{N}$ & $\mathrm{N}$ & $\mathrm{N}$ & $\mathrm{N}$ & $\mathrm{N}$ & $\mathrm{N}$ & $\mathrm{Y}$ & $\mathrm{Y}$ & $\mathrm{N}$ & $\mathrm{Y}$ & 4 \\
\hline Chiaia et $\mathrm{al}^{36}$ & $\mathrm{Y}$ & $\mathrm{N}$ & $\mathrm{N}$ & $\mathrm{N}$ & $\mathrm{N}$ & $\mathrm{N}$ & $\mathrm{N}$ & $\mathrm{Y}$ & $\mathrm{Y}$ & $\mathrm{N}$ & $\mathrm{Y}$ & 4 \\
\hline
\end{tabular}

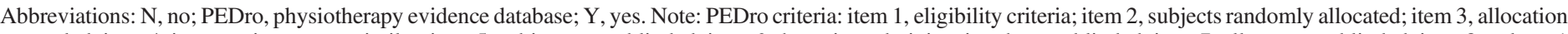

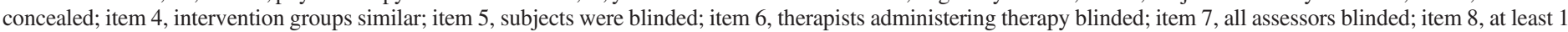

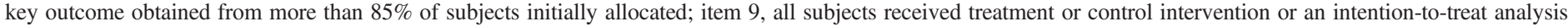

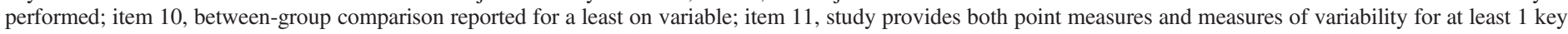
outcome. 


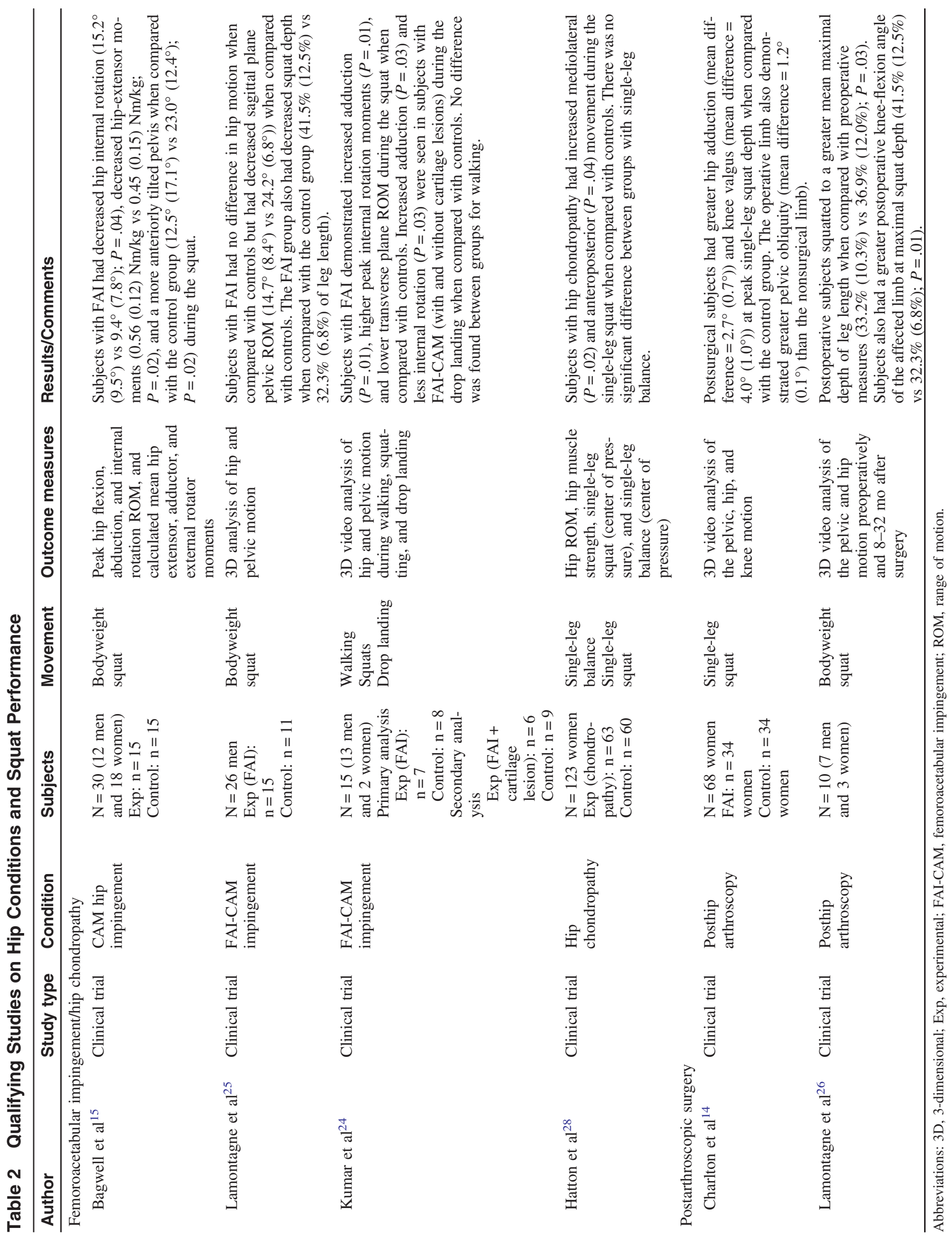




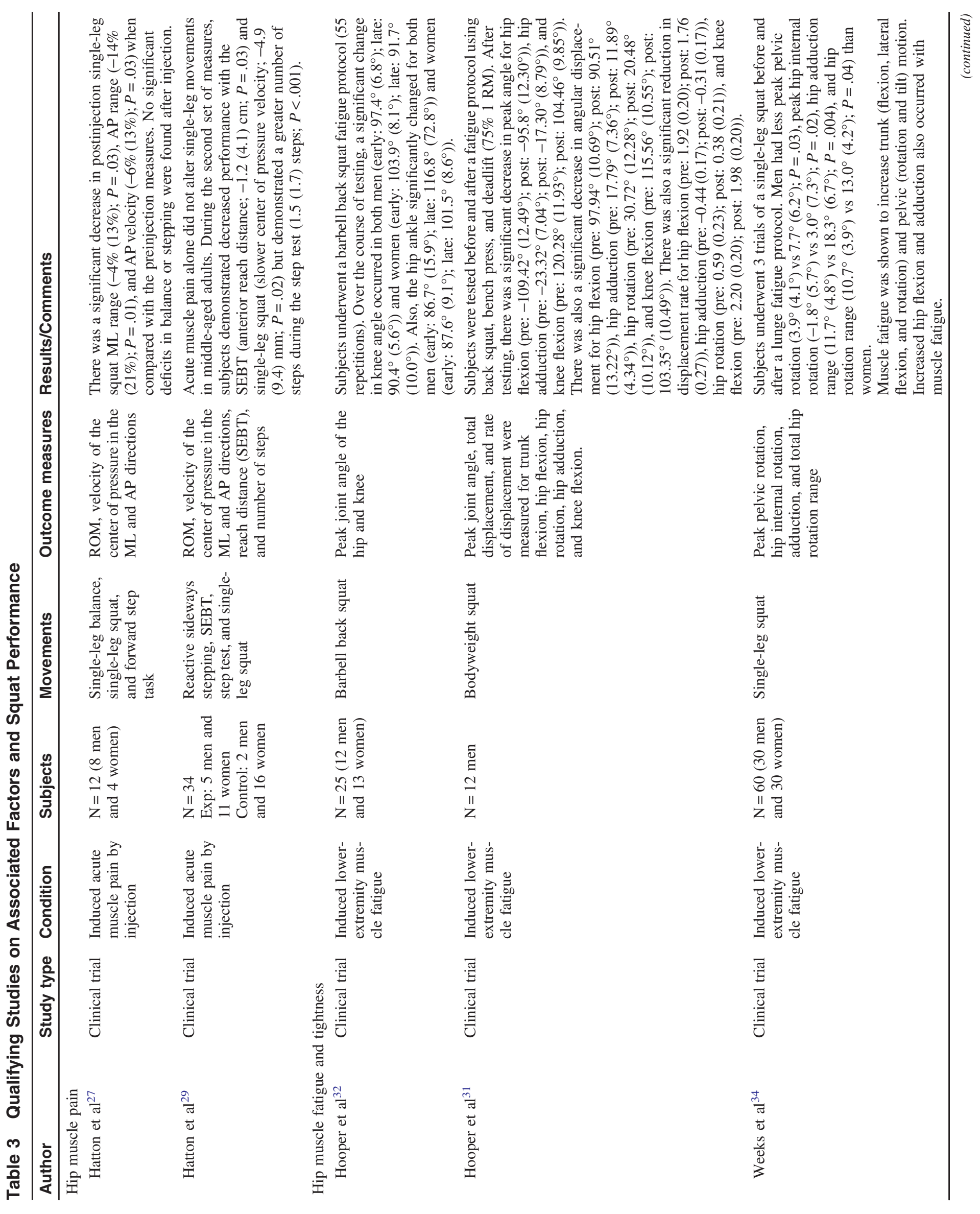




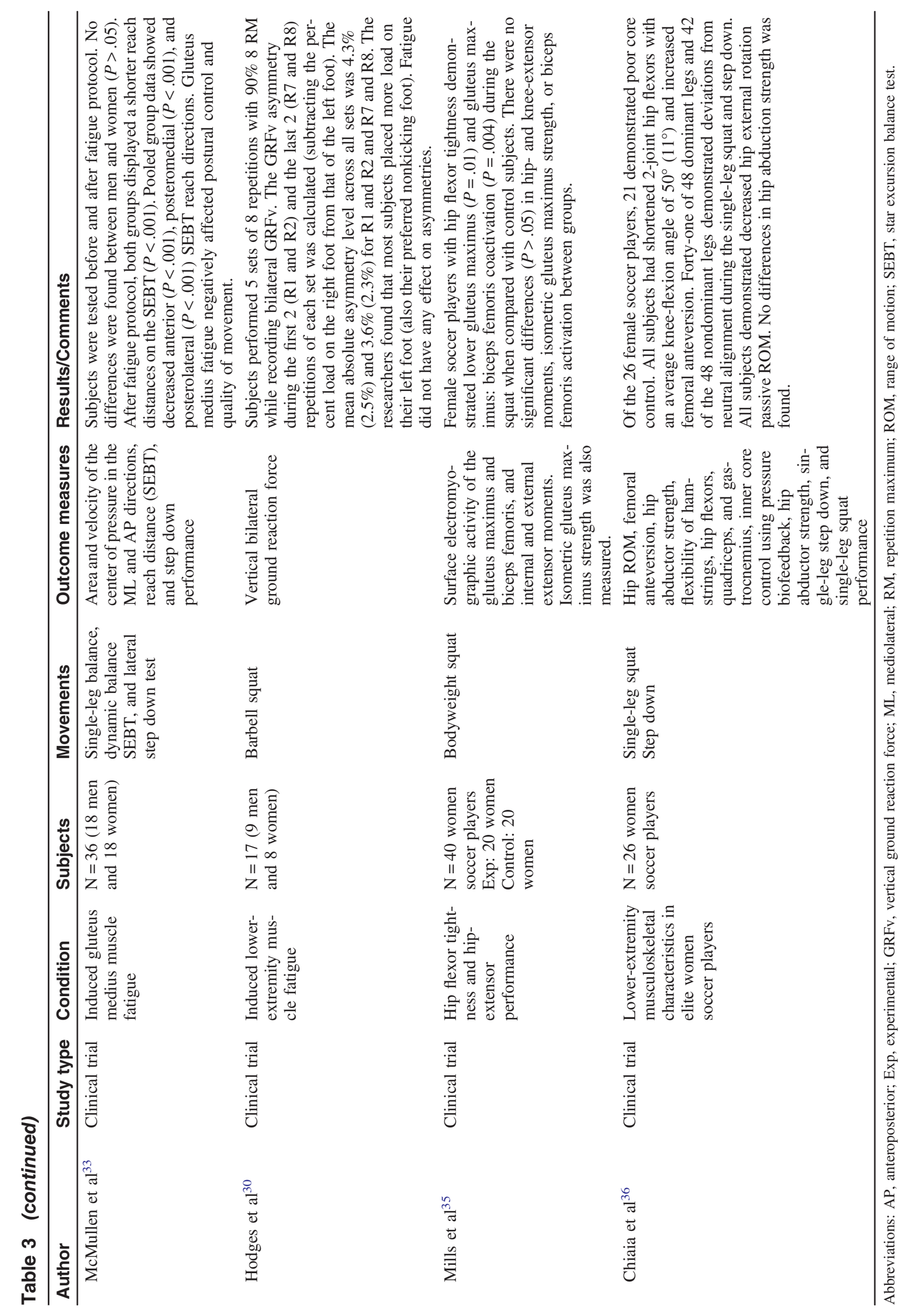




\section{Hip Chondropathy}

$\mathrm{HC}$ is classified as disease of the articular cartilage and is often associated with early hip osteoarthritis. ${ }^{37}$ This condition is commonly seen in young to middle-aged individuals below the age of 60 years. ${ }^{38} \mathrm{HC}$ can have an influence on squat performance in these individuals. Hatton et $\mathrm{al}^{28}$ examined the single-leg squat (eyes open) and single-leg balance tasks (eyes closed) in 123 women subjects (mean age $=37.5(10.6)$ y). Group 1 consisted of 63 subjects diagnosed with $\mathrm{HC}$ and group 2 consisted of 60 control subjects. The researchers analyzed center of pressure movement in the mediolateral (ML) and anteroposterior (AP) directions, hip ROM, and muscle strength. Subjects stood on a Nintendo Wii balance board for the center of pressure measures. Upon completion, the researchers found that subjects with $\mathrm{HC}$ had increase ML $(P=.02)$ and AP movement $(P=.04)$ during the single-leg squat when compared with controls. There was no significant difference between groups with single-leg balance. The results suggest that single-leg squat performance is reduced in individuals who underwent hip chondroplasty. ${ }^{28}$

\section{Hip Arthroscopic Surgery}

Some individuals who have undergone hip arthroscopy to correct FAI may have more ROM during the squat but may have decreased motor control. Charlton et $\mathrm{al}^{14}$ examined pelvic and hip kinematics during a bodyweight maximal depth single-leg squat in a group of 68 women subjects (mean age $=34.9(12.2)$ y). Group 1 consisted of 34 subjects 1-2 years post unilateral hip arthroscopy and group 2 consisted of 34 matched healthy controls. The researcher analyzed pelvic, hip, and knee motion during the squat using video analysis. Upon completion, the researchers found greater hip adduction (mean difference $=2.7^{\circ}$ ) and knee valgus (mean difference $=$ $4.0^{\circ}$ ) at peak squat depth when compared with the control group. The operative limb also demonstrated greater pelvic obliquity than the nonsurgical limb. ${ }^{14}$ Lamontagne et $\mathrm{al}^{26}$ analyzed the presurgical and postsurgical pelvic and hip kinematics during a bodyweight maximal depth squat in 10 subjects with FAI-CAM impingement (7 men and 3 women; mean age $=24$ (7.2) y). The researchers analyzed hip and pelvic motion during the squat using 3D analysis preoperatively and 8 to 32 months after surgery. Upon completion, the researchers found that postoperatively the subjects affected limb demonstrated greater squat depth $(P=.03)$, and greater peak hip- and knee-flexion angles $(P=.01)$ and ankle dorsiflexion angles $(P=.048)$ when compared with preoperative measures. ${ }^{26}$

\section{Hip Muscle Pain}

Hip muscle pain is a common symptom in many hip conditions and often can have a negative effect on exercise performance. ${ }^{8}$ Two studies examined dynamic single-limb movements and balance after induced hip muscle pain. Hatton et $\mathrm{al}^{27}$ examined 3 single-leg movements after injecting the gluteus medius with a hypertonic saline solution in a group of 12 younger adult subjects ( 8 men and 4 women; mean age $=27.1$ (3) y). The movements included singleleg standing (eyes closed), single-leg squat (eyes open), and a forward step (eyes open). Outcome measures included range of movement and the velocity of the center of pressure in the ML and AP directions. The movements and measures were performed before and after injection. Upon completion, the researchers found that during the single-leg squat, there was a significant decrease in postinjection range $(P \leq .01)$ and velocity $(P=.03)$ in the ML and
AP directions when compared with the preinjection measures. No deficits in balance or stepping were found after injection. ${ }^{27}$

Hatton et $\mathrm{al}^{29}$ conducted another study examining 4 dynamic single-leg movements in a group of 34 middle-aged subjects assigned to 2 groups: experimental (11 women and 5 men; age = 50.5 (3.4) y) and control (16 women and 2 men; age $=51.6(4.6)$ y). The experimental group received an injection in the gluteus medius with a hypertonic saline solution. The movements analyzed included reactive sideways stepping, star excursion balance test (SEBT), step test, and single-leg squat. Outcome measures included range of movement, velocity of the center of pressure in the ML and AP directions for the reactive sidestepping and squat. Reach distance was calculated for the SEBT and number of completed steps for the step test. The movements and measures were performed before and after injection for the experimental group or a 5-minute rest for the control group. Upon completion, the researchers found that acute muscle pain alone did not alter single-leg movements in middle-aged adults. During the second set of measures, subjects demonstrated decreased performance (irrespective of exposure to hip muscle pain) with the SEBT (anterior reach distance; $P=.03$ ) and single-leg squat (slower center of pressure velocity; $P=.02$ ) but demonstrated a greater number of steps during the step test $(P<.001)$ when compared with baseline measures. Factors other than pain may have an influence on single-leg performance and balance in middle-aged adults.

\section{Hip Muscle Fatigue}

Athletes and active adults with current hip conditions may experience muscle fatigue due to compensatory movements from pain or weakness. ${ }^{12,39}$ Researchers have looked at the effects of induced muscle fatigue on the squat motion. Hooper et $\mathrm{al}^{31}$ examined the effects of lower-extremity muscle fatigue on barbell back squat performance. The researchers tested 25 subjects (12 men and 13 women; mean age $=24.2$ (4) y) using a repetitive barbell back squat fatigue protocol (55 repetitions). The researchers conducted a 2-dimensional analysis on hip and knee joint motion during the repetitive movement. Upon completion, the researchers found that at the early stages of the protocol sagittal plane hip and knee angles decreased in both sexes. This was demonstrated by a greater forward trunk lean and less knee flexion by the subjects. Thus, subjects compensated for their fatigue by leaning forward more and not squatting as low during the movement. ${ }^{31}$

Hooper et $\mathrm{al}^{32}$ conducted another study examining the effects of lower-extremity muscle fatigue on body weight squat performance. The researchers tested 12 male subjects (mean age $=24$ (4.2) y) with resistance training experience using an exercise fatigue protocol $(75 \% 1$ repetition maximum) using the back squat, bench press, and deadlift. The subjects began by performing each lift (as quickly as possible) for 10 repetitions and then reduced the number consecutively until they reached 1 repetition of each movement. The researchers conducted a 3D analysis of the subjects performing 5 bodyweight squats before and after the fatigue protocol. Peak joint angle, total displacement, and rate of displacement were measured for trunk flexion, hip flexion, hip rotation, hip adduction, and knee flexion. Upon completion, the researchers found a significant decrease in peak angle for hip flexion, adduction, and knee flexion after the fatigue protocol. There was also a significant decrease in angular displacement and rate of displacement for hip flexion, adduction, rotation, and knee flexion. The results indicated that when fatigued subjects squatted with less hip 
flexion, hip adduction, and knee flexion and at a slower rate for hip flexion, hip adduction, hip rotation, and knee flexion. ${ }^{32}$

Weeks et $\mathrm{al}^{34}$ examined the effects of lower-extremity muscle fatigue and sex differences on single-leg squat performance in 60 subjects (30 men and 30 women; mean age $=25.3(4.3) \mathrm{y}$ ). The researchers conducted 3 trials of the single-leg squat before and after the fatigue protocol. The fatigue protocol began with a maximal vertical jump height measurement followed by repeated lunges (3 sets 20) and retesting of the jump height. This sequence was repeated with an additional 10 lunges until jump height diminished by $20 \%$ or the participant could no longer continue to perform lunges. During the single-leg squat, the researchers measured peak pelvic rotation, hip internal rotation, hip adduction, and total hip rotation ROM using video motion analysis. Upon completion, the researchers found that males had less peak ROM in all motions $(P \leq .04)$ when compared with females. Muscle fatigue was shown to significantly increase $(P \leq .05)$ trunk (flexion, lateral flexion, and rotation) and pelvic obliquity (toward stance leg), and pelvic rotation (away from stance leg). Increased hip flexion and adduction also occurred with muscle fatigue. ${ }^{34}$

McMullen et $\mathrm{al}^{33}$ examined the effects of a gluteus medius fatigue protocol on dynamic single-leg movements and balance in 38 healthy subjects ( 18 men and 18 women; mean age $=22(3.4) \mathrm{y}$ ). The fatigue protocol included repeated dominant leg sidelying hip abduction until a $15 \%$ shift in electromyographic median frequency of the gluteus medius was reached. The movements analyzed included single-leg static balance, dynamic balance, SEBT, and lateral step down test. Outcome measures included area and velocity of the center of pressure in the ML and AP directions, reach distance (SEBT), and step down performance. The movements and measures were performed before and after the fatigue protocol. Upon completion, the researchers found that gluteus medius fatigue negatively affected postural control and quality of movement $(P \leq .05)$. No differences were found between men and women $(P>.05)$. It is important to note that the lateral step down is a common version of the single-leg squat and produces similar lower-extremity kinematics. ${ }^{40}$

Hodges et $\mathrm{al}^{30}$ examined the presence of fatigue-induced asymmetries during the squat movement in 17 subjects ( 9 men and 8 women; mean age $=22.3(2.5) \mathrm{y}$ ). The fatigue protocol included 5 sets of 8 repetitions at $90 \% 8$ repetitions maximum. The researchers analyzed bilateral vertical ground reaction during the movement. Upon completion, the researchers found that subjects demonstrated consistent asymmetries (weight shift toward the right or left side) throughout the movement. Fatigue did not have any effect on asymmetries. ${ }^{30}$

\section{Hip Muscle Tightness}

Lower-extremity muscle tightness is a common concern among fitness professionals as it may be linked to various hip conditions and may affect performance. ${ }^{8}$ The research on the effects of hip muscle tightness on squat performance is still emerging. Mills et $\mathrm{al}^{35}$ examined the association between hip flexor muscle tightness and hip-extensor muscle performance during a squat activity. Forty female soccer players (mean age $=19.7$ (1) y) participated and were placed into 2 groups: (1) tight hip flexors (confirmed via Thomas test) and (2) control group. The researcher analyzed surface electromyographic activity of the gluteus maximus and biceps femoris, and internal and external extensor moments during a bodyweight squat. Isometric gluteus maximus strength was also measured. Upon completion, the researchers found that female soccer players with hip flexor tightness demonstrated lower gluteus maximus activation $(P=.01)$ and gluteus maximus-biceps femoris coactivation $(P=.004)$ during the squat when compared with control subjects. There were no significant differences in hipand knee-extensor moments, isometric gluteus maximus strength, or biceps femoris activation between groups. ${ }^{35}$

Chiaia et $\mathrm{al}^{36}$ conducted a study to identify lower-extremity musculoskeletal characteristics among elite female soccer players. Twenty-six female adult professional soccer players (mean age of 25 y [range: 21-32 y]) participated in the study. The researcher analyzed bilateral hip passive ROM; femoral anteversion; flexibility of hamstrings, hip flexors, quadriceps, and gastrocnemius; inner core control using pressure biofeedback; hip abductor strength; single-leg step down; and single-leg squat. ${ }^{36}$ Upon completion, the researcher found that 21 of 26 subjects demonstrated poor core control. All subjects had shortened 2-joint hip flexors and increased femoral anteversion. Forty-one of 48 dominant legs and 42 of 48 nondominant legs demonstrated deviations from neutral alignment during the single-leg squat and step down. All subjects demonstrated decreased hip external rotation passive ROM. No differences in hip abduction strength were found. ${ }^{36}$

\section{Discussion}

The purpose of this systematic review was to appraise the current literature on the effects of hip musculoskeletal conditions and associated factors, such as hip muscle pain, fatigue, and tightness, on squat performance. To the author's knowledge, a collective appraisal of the literature studying the association of these topics and squat performance has not been published as research has tended to focus on the association of hip muscle weakness and decreased bilateral ${ }^{16-20}$ and single-leg squat ${ }^{21-23}$ performance.

The study of the association between hip conditions and squat performance is still emerging. The current research is limited to intraarticular conditions, such as FAI and HC. The available research suggests that individuals with FAI-CAM type may demonstrate decreased pelvic motion, increased anterior pelvic tilt, decreased squat depth, and increased femoral adduction and internal rotation with bilateral squats. ${ }^{15,24,25}$ Therefore, allied health and fitness professionals should allow the individual to work in a painfree ROM. This may include reducing the depth of the squat to avoid the impinging. As Lamontagne et $\mathrm{al}^{25}$ found, FAI-CAM decreased sagittal plane motion of the pelvis during the squat. Therefore, squat ROM may need to be reduced for these individuals. Additionally, including hip extensor, hip external rotator, and hip abductor activation and strengthening drills, such as glute bridges and clamshells, into the FAI-CAM program as part of a dynamic warm-up is suggested to potentially offset or reduce the risk of damage associated with the less than ideal joint motion. Individuals with $\mathrm{HC}$ may also demonstrate increased $\mathrm{ML}$ and $\mathrm{AP}$ movements during a single-leg squat. ${ }^{28} \mathrm{HC}$ is often associated with early stages of osteoarthritis; therefore, pain-free ROM must be emphasized. Allied health and fitness professionals should consider that these individuals with intraarticular pathology may be symptomatic and may present with other associated factors, such as pain, fatigue, and muscle tightness. ${ }^{8,12}$

Individuals with FAI or chondropathy may undergo hip arthroscopy for surgical correction. The current research on postsurgical squat performance is mixed. Individuals after hip arthroscopy were able to demonstrate greater postoperative squat depth when compared with preoperative measures. ${ }^{26}$ However, they also had decreased performance with the single-leg squat demonstrated 
by increased femoral adductor and internal rotation. ${ }^{14}$ Allied health and fitness professionals should consider that, although individuals who undergo hip arthroscopic surgery may have more ROM during a squat, they may still have motor control deficits as indicated by compensatory movement through the knee, hip, and pelvis. ${ }^{14}$ Basic information on whether or not an arthroscopic procedure has taken place should be obtained while gathering preparticipation information. This will help the professional to better identify the root cause of compensations and to design a more effective overall program.

As far as hip muscle pain, the current research suggests that hip muscle pain may cause decreased single-leg squat performance in younger individuals ${ }^{27}$ but have no effect on middle-aged adults. ${ }^{29}$ Previous research has supported the notion that pain may alter the motor cortex and negatively effective movement. ${ }^{41}$ Therefore, professionals need to consider that the younger individuals decreased single-leg squat performance may be a direct result of the pain. This pain may need to be initially evaluated by the properly licensed clinician.

The middle-aged adult may demonstrate decreased performance on the single-leg squat due to factors other than induced muscle pain. ${ }^{29}$ For hip muscle fatigue, the current literature suggests that individuals may demonstrate compensatory movements with the barbell back squat, ${ }^{32}$ bodyweight squat, ${ }^{31}$ and single-leg squat $^{33,34}$ when the lower-extremity muscles are fatigued. As this review has indicated, if the professional identifies compensatory movements during the squat, such as excessive trunk flexion, it may be associated with fatigue of trunk extenders. A previously fatigued gluteus medius may also result in less postural control during single-leg activities.

For hip muscle tightness, the research is emerging with only 2 investigations suggesting that hip-extensor function is decreased during the squat in individuals with tight hip flexors. ${ }^{35,36}$ It is important to note that only female soccer players were investigated, which limits the generalizability to this population. ${ }^{35,36}$ As the researchers ${ }^{35,36}$ found, tight hip flexors inhibited the hip extensors. The hip extensors are a primary muscle for decelerating during the descent and for accelerating the ascent. If the prime move is not able to contribute to the majority of the movement, then secondary muscles will increase activity. This process, known as synergistic dominance, is a contributor to common musculoskeletal injuries, such as hamstring injury, groin injury, and low back pain. ${ }^{42}$ Much of the movement impairment caused by tight muscles and muscles with less than optimal strength can be identified in complex motions, such as a squat. For example, weak gluteus maximus has been associated with increased anterior pelvic tilt, ${ }^{42}$ and weak gluteus medius associated with increased knee valgus. ${ }^{42}$ Thus, the professional armed with the knowledge of tight hip flexors reducing squat performance may also be more likely to design more effective programs.

The allied health and fitness professionals should consider many factors that may affect the performance of the squat, such as FAI and HC. ${ }^{14,15}$ Associated factors, such as muscle pain, fatigue, and tightness, may accompany hip conditions and can influence squat performance. ${ }^{8,12}$

\section{Limitations}

Several limitations warrant discussion for this review. The main limitation is the paucity and heterogeneity of evidence surrounding the association of hip musculoskeletal conditions and associated factors and squat performance. The qualifying studies have varied methods, sample sizes, and outcome measures that prevent a direct comparison among studies. Another limitation is that the qualified studies represent only the specific search criteria that included only English-language publications and that may not have represented the available evidence in non-English peer-reviewed publications. Also, muscle strength deficits and total joint replacements were not considered for this analysis as they both have a large, existing body of literature. An analysis of these topics is scheduled for a future publication.

\section{Recommendations and Future Research}

The squat is a fundamental movement for athletic performance and activities of daily living. The existing literature provides some insight into intraarticular conditions and associated factors, such as muscle pain, fatigue, and tightness, and their influence on squat performance. The results of this appraisal suggest that intraarticular pathologies and the associated factors can negatively affect squat performance. Therefore, a client with an FAI may have decreased squat performance due to joint restrictions and associated factors, such as pain, fatigue, and tightness. ${ }^{12}$ Allied health and fitness professionals should consider these findings when designing and prescribing an individualized exercise program involving squats for these individuals. The research on this topic is sparse and is limited to the current findings. Allied health and fitness professionals must consider this when integrating such findings into their practice. Future research should focus on the association of other intraarticular and extraarticular conditions and squat performance.

\section{Conclusion}

The research examining the association between hip musculoskeletal conditions and associated factors on squat performance is still emerging. This is the first systematic review to appraise the evidence on these topics. These results provide a starting point for allied health and fitness professionals to better understand this topic and to help guide safe exercise prescription for these individuals. Future research should focus on other hip conditions and their influence on squat performance.

\section{References}

1. Speirs DE, Bennett MA, Finn CV, Turner AP. Unilateral vs. bilateral squat training for strength, sprints, and agility in academy rugby players. J Strength Cond Res. 2016;30(2):386-392. PubMed doi:10. 1519/JSC.0000000000001096

2. Kivlan BR, Martin RL. Functional performance testing of the hip in athletes: a systematic review for reliability and validity. Int J Sports Phys Ther. 2012;7(4):402-412. PubMed

3. Ugalde V, Brockman C, Bailowitz Z, Pollard CD. Single leg squat test and its relationship to dynamic knee valgus and injury risk screening. PM R. 2015;7(3):229-235; quiz 235. PubMed doi:10.1016/j.pmrj. 2014.08.361

4. Clifton DR, Grooms DR, Onate JA. Overhead deep squat performance predicts functional movement screen score. Int J Sports Phys Ther. 2015;10(5):622-627. PubMed

5. Mauntel TC, Post EG, Padua DA, Bell DR. Sex differences during an overhead squat assessment. J Appl Biomech. 2015;31(4):244-249. PubMed doi:10.1123/jab.2014-0272

6. Macrum E, Bell DR, Boling M, Lewek M, Padua D. Effect of limiting ankle-dorsiflexion range of motion on lower extremity kinematics and 
muscle-activation patterns during a squat. $J$ Sport Rehabil. 2012;21(2):144-150. PubMed doi:10.1123/jsr.21.2.144

7. Dill KE, Begalle RL, Frank BS, Zinder SM, Padua DA. Altered knee and ankle kinematics during squatting in those with limited weightbearing-lunge ankle-dorsiflexion range of motion. J Athl Train. 2014;49(6):723-732. PubMed doi:10.4085/1062-6050-49.3.29

8. Cheatham SW, Kolber MJ. Orthopedic Management of the Hip and Pelvis. Philadelphia, PA: Elsevier-Health Sciences Division; 2015.

9. Larkin B. Epidemiology of hip and pelvis injury. In: Seidenberg P, Bowen JD, eds. The Hip and Pelvis in Sports Medicine and Primary Care. New York, NY: Springer; 2010:1-7.

10. Boyd KT, Peirce NS, Batt ME. Common hip injuries in sport. Sports Med. 1997;24(4):273-288. PubMed doi:10.2165/00007256199724040-00005

11. DeAngelis NA, Busconi BD. Assessment and differential diagnosis of the painful hip. Clin Orthop Relat Res. 2003;406:11-18. doi:10. 1097/00003086-200301000-00005

12. Cheatham SW, Enseki KR, Kolber MJ. The clinical presentation of individuals with femoral acetabular impingement and labral tears: a narrative review of the evidence. J Bodyw Mov Ther. 2016;20(2): 346-355. PubMed doi:10.1016/j.jbmt.2015.10.006

13. Diamond LE, Dobson FL, Bennell KL, Wrigley TV, Hodges PW, Hinman RS. Physical impairments and activity limitations in people with femoroacetabular impingement: a systematic review. Br J Sports Med. 2015;49(4):230-242. PubMed doi:10.1136/bjsports-2013093340

14. Charlton PC, Bryant AL, Kemp JL, Clark RA, Crossley KM, Collins NJ. Single-leg squat performance is impaired 1 to 2 years after hip arthroscopy. PM R. 2016;8(4):321-330. PubMed doi:10.1016/j.pmrj. 2015.07.004

15. Bagwell JJ, Snibbe J, Gerhardt M, Powers CM. Hip kinematics and kinetics in persons with and without cam femoroacetabular impingement during a deep squat task. Clin Biomech. 2016;31:87-92. doi:10. 1016/j.clinbiomech.2015.09.016

16. Crossley KM, Zhang WJ, Schache AG, Bryant A, Cowan SM. Performance on the single-leg squat task indicates hip abductor muscle function. Am J Sports Med. 2011;39:866-873. PubMed doi:10.1177/0363546510395456

17. Mauntel TC, Begalle RL, Cram TR, et al. The effects of lower extremity muscle activation and passive range of motion on single leg squat performance. J Strength Cond Res. 2013;27:1813-1823. PubMed doi:10.1519/JSC.0b013e318276b886

18. Stickler L, Finley M, Gulgin H. Relationship between hip and core strength and frontal plane alignment during a single leg squat. Phys Ther Sport. 2015;16(1):66-71. PubMed doi:10.1016/j.ptsp.2014. 05.002

19. Trulsson A, Miller M, Hansson G-Å, Gummesson C, Garwicz M. Altered movement patterns and muscular activity during single and double leg squats in individuals with anterior cruciate ligament injury. BMC Musculoskelet Disord. 2015;16(1):28. doi:10.1186/s12891015-0472-y

20. Claiborne TL, Armstrong CW, Gandhi V, Pincivero DM. Relationship between hip and knee strength and knee valgus during a single leg squat. J Appl Biomech. 2006;22(1):41-50. PubMed doi:10.1123/ jab.22.1.41

21. Hollman JH, Galardi CM, Lin IH, Voth BC, Whitmarsh CL. Frontal and transverse plane hip kinematics and gluteus maximus recruitment correlate with frontal plane knee kinematics during single-leg squat tests in women. Clin Biomech. 2014;29(4):468-474. doi:10.1016/ j.clinbiomech.2013.12.017

22. Raisanen A, Pasanen K, Krosshaug T, Avela J, Perttunen J, Parkkari J. Single-leg squat as a tool to evaluate young athletes' frontal plane knee control. Clin J Sport Med. 2016;26(6):478-482. PubMed doi:10. 1097/JSM.0000000000000288

23. Nguyen AD, Shultz SJ, Schmitz RJ, Luecht RM, Perrin DH. A preliminary multifactorial approach describing the relationships among lower extremity alignment, hip muscle activation, and lower extremity joint excursion. $J$ Athl Train. 2011;46(3):246-256. PubMed doi:10.4085/1062-6050-46.3.246

24. Kumar D, Dillon A, Nardo L, Link TM, Majumdar S, Souza RB. Differences in the association of hip cartilage lesions and cam-type femoroacetabular impingement with movement patterns: a preliminary study. PM R. 2014;6(8):681-689. PubMed doi:10.1016/j.pmrj. 2014.02.002

25. Lamontagne M, Kennedy MJ, Beaule PE. The effect of cam FAI on hip and pelvic motion during maximum squat. Clin Orthop Relat Res. 2009;467(3):645-650. PubMed doi:10.1007/s11999-0080620-X

26. Lamontagne M, Brisson N, Kennedy MJ, Beaule PE. Preoperative and postoperative lower-extremity joint and pelvic kinematics during maximal squatting of patients with cam femoro-acetabular impingement. J Bone Joint Surg Am. 2011;93(suppl 2):40-45. doi:10.2106/ JBJS.J.01809

27. Hatton AL, Crossley KM, Hug F, et al. Acute experimental hip muscle pain alters single-leg squat balance in healthy young adults. Gait Posture. 2015;41(4):871-876. PubMed doi:10.1016/j.gaitpost. 2015.02.013

28. Hatton AL, Kemp JL, Brauer SG, Clark RA, Crossley KM. Impairment of dynamic single-leg balance performance in individuals with hip chondropathy. Arthritis Care Res. 2014;66(5):709-716. doi:10.1002/acr.22193

29. Hatton AL, Hug F, Chen SH, Reid C, Sorensen NA, Tucker K. The effects of acute experimental hip muscle pain on dynamic singlelimb balance performance in healthy middle-aged adults. Gait Posture. 2016;50:201-206. PubMed doi:10.1016/j.gaitpost.2016. 09.007

30. Hodges SJ, Patrick RJ, Reiser RF 2nd. Effects of fatigue on bilateral ground reaction force asymmetries during the squat exercise. $J$ Strength Cond Res. 2011;25(11):3107-3117. PubMed doi:10.1519/ JSC.0b013e318212de7b

31. Hooper DR, Szivak TK, Comstock BA, et al. Effects of fatigue from resistance training on barbell back squat biomechanics. J Strength Cond Res. 2014;28(4):1127-1134. PubMed

32. Hooper DR, Szivak TK, Distefano LJ, et al. Effects of resistance training fatigue on joint biomechanics. J Strength Cond Res. 2013; 27(1):146-153. PubMed doi:10.1519/JSC.0b013e31825390da

33. McMullen KL, Cosby NL, Hertel J, Ingersoll CD, Hart JM. Lower extremity neuromuscular control immediately after fatiguing hipabduction exercise. J Athl Train. 2011;46(6):607-614. PubMed doi:10.4085/1062-6050-46.6.607

34. Weeks BK, Carty CP, Horan SA. Effect of sex and fatigue on single leg squat kinematics in healthy young adults. BMC Musculoskelet Disord. 2015;16:271. PubMed doi:10.1186/s12891-0150739-3

35. Mills M, Frank B, Goto S, et al. Effect of restricted hip flexor muscle length on hip extensor muscle activity and lower extremity biomechanics in college-aged female soccer players. Int J Sports Phys Ther. 2015;10(7):946-954. PubMed

36. Chiaia TA, Maschi RA, Stuhr RM, et al. A musculoskeletal profile of elite female soccer players. HSS J. 2009;5(2):186-195. PubMed doi:10.1007/s11420-009-9108-9

37. Kemp JL, Makdissi M, Schache AG, Pritchard MG, Pollard TC, Crossley KM. Hip chondropathy at arthroscopy: prevalence and relationship to labral pathology, femoroacetabular impingement 
and patient-reported outcomes. Br J Sports Med. 2014;48(14): 1102-1107. PubMed doi:10.1136/bjsports-2013-093312

38. Kemp JL, Makdissi M, Schache AG, Pritchard MG, Crossley KM. Hip chondropathy is prevalent at arthroscopy and is associated with co-existing pathology, but not patient reported outcomes. Osteoarthritis Cartilage. 2013;21:S138. doi:10.1016/j.joca.2013.02.293

39. Cheatham SW, Kolber MJ. Rehabilitation after hip arthroscopy and labral repair in a high school football athlete: a 3.6 year follow-up with insight into potential risk factors. Int J Sports Phys Ther. 2015;10(4):530-539. PubMed
40. Lewis CL, Foch E, Luko MM, Loverro KL, Khuu A. Differences in lower extremity and trunk kinematics between single leg squat and step down tasks. PLoS ONE. 2015;10(5):e0126258. PubMed doi:10. 1371/journal.pone.0126258

41. Farina S, Tinazzi M, Le Pera D, Valeriani M. Pain-related modulation of the human motor cortex. Neurol Res. 2003;25(2):130-142. PubMed doi:10.1179/016164103101201283

42. Clark M, Lucett S, Sutton BG; National Academy of Sports Medicine. NASM Essentials of Corrective Exercise Training. Burlington, MA: Jones \& Bartlett Learning; 2014. 